\title{
A GENERAL DESIGN FOR ENERGY TEST PROCEDURES
}

\author{
Alan Meier \\ Lawrence Berkeley National Laboratory \\ June 2000
}

\begin{abstract}
Appliances are increasingly controlled by microprocessors. Unfortunately, energy test procedures have not been modified to capture the positive and negative contributions of the microprocessor to the appliance's energy use. A new test procedure is described, which captures both the mechanical and logical features present in many new appliances. We developed an energy test procedure for refrigerators that incorporates most aspects of the proposed new approach. Some of the strengths and weaknesses of the new test are described.
\end{abstract}

\section{WHY ARE NEW ENERGY TEST PROCEDURES NEEDED?}

The technologies employed in major appliances are undergoing a major transformation. In the past, most aspects of an appliance's operation were controlled by the user. New appliances, however, have microprocessor controls, which may adjust the appliance's operation without any action by (or even knowledge of) the user. The microprocessor can gather information through sensors or from memory of previous cycles to select an operating strategy that results in enhanced amenities or services to the user (Meier 1997; Meier 1998).

This trend has the potential to save energy, water, and other resources in many different ways. For example in washing machines, sensors may measure the weight of clothing and the extent of soiling. The microprocessor will use this information to select the minimum amount of water and detergent to achieve clean clothes. Microprocessors can also control variable-speed motors in air conditioners and refrigerators; this will allow better temperature regulation, dehumidification, often with less energy than traditional approaches.

Unfortunately the energy savings from microprocessor controls are not fully captured in the present energy test procedures. Some omissions in the present tests are that they:

- Fail to include part-load conditions (less than full loads in washing machines, cooling or heating at less than steady-state output, etc.)

- Ignore learning capability from previous cycles 
- Ignore sensing special conditions for service (such as level of soiling and type of fabric)

- Fail to recognize communication between the appliance and a network (including the internet)

In some cases these omissions lead to only a small discrepancy between the laboratory measurements and actual use, but this discrepancy is likely to grow as microprocessors become more sophisticated.

At the same time, some manufacturers are programming the microprocessors in appliances to recognize when the appliances are being tested. When the unique test conditions are identified, the microprocessor modifies performance such that it uses less energy than it would under ordinary conditions.

This paper outlines a general approach to energy tests for this new generation of appliances. These tests aim to assess the integrated performance of both the appliance's mechanical features (the "hardware") and its logical features (the "software").

\section{ELEMENTS OF THE NEW APPROACH}

In the new approach, the test procedure consists of "hardware tests" and "software tests." The results of these tests are inputs to a model that simulates the appliance's energy use. The simulation model programmed to predict energy use in any conditions requested by the user.

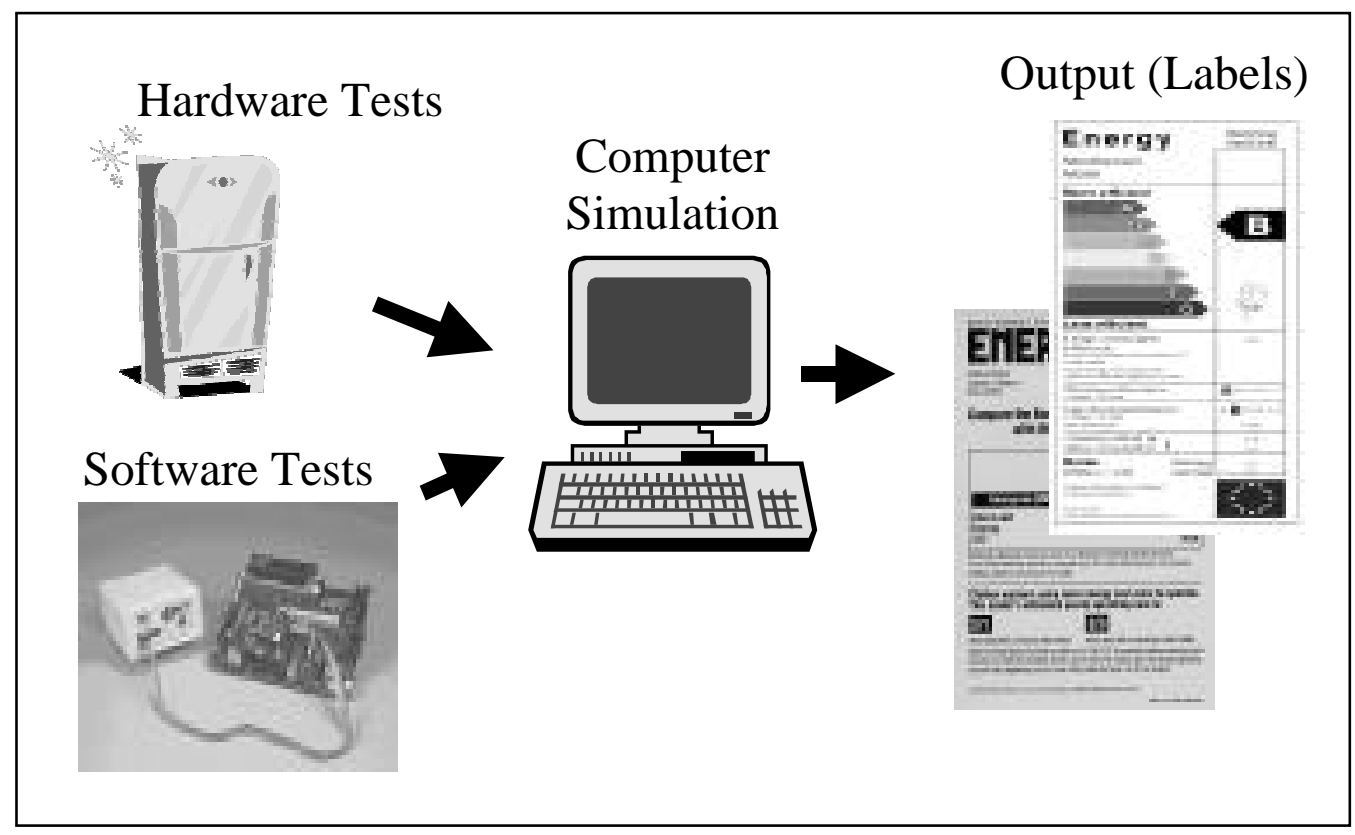

Figure 1. Elements of the new energy test procedure. 
The test conditions may be those specified by any of the major existing test procedures (ISO, IEC, DOE or JIS). This predicted energy use is the information needed to demonstrate compliance with energy efficiency regulations or to prepare an energy label. Figure 1 shows the flow of information in the proposed test procedure.

The hardware tests differ from present tests because the goal is to extract performance parameters, such as the COP, the water consumption, the heat loss coefficient, etc., which can be used to simulate the appliance's performance. The software tests are a new concept but are similar to "reverse engineering", which is often performed by electronics manufacturers and others in order to understand a competitor's product. In the following sections, each of these elements is described in detail for the refrigerator test procedure.

\section{APPLICATION OF GENERAL APPROACH TO REFRIGERATOR TEST PROCEDURES}

This approach differs significantly from existing test procedures, although certain aspects are similar to specific tests. For example, the U.S. DOE test procedure for central air conditioners uses a simple hardware test to derive performance parameters that are used to calculate a seasonal performance value (Domanski 1989). The original Japanese refrigerator test (Japanese Standards Association 1979) combines results from a "winter" and a "summer" condition to derive a weighted result. This is a crude simulation model that produces energy consumption in an "average" condition. However, no test has combined all aspects of the proposed test procedure. For this reason, we applied the general approach to a specific appliance: the refrigerator. The goal was to learn what kinds of problems might arise. Some of the results are described below.

\section{Hardware Tests}

The goal of the hardware tests is to collect overall mechanical efficiency parameters for the appliance. An example of the kind of efficiency parameters collected in the hardware tests for a refrigerator is shown in Figure 2.

Four separate hardware tests are performed. The shell test seeks to measure the overall heat loss coefficient of the refrigerator's box. The door test measures the heat gain caused by door openings (and thus captures certain aspects of the refrigerator's geometry). The COP test measures the efficiency of heat extraction over a range of temperatures. Finally, the load test captures the refrigerator's response to internal sensible and latent loads. These parameters are entered in the simulation model to help predict energy consumption of the refrigerator.

The hardware tests need to satisfy two conflicting requirements. The simulation model needs detailed data in order to be properly calibrated. On the other hand, 
hardware tests are expensive and have limited precision. The challenge is to devise hardware tests that are both simple to perform and yield sufficient information to

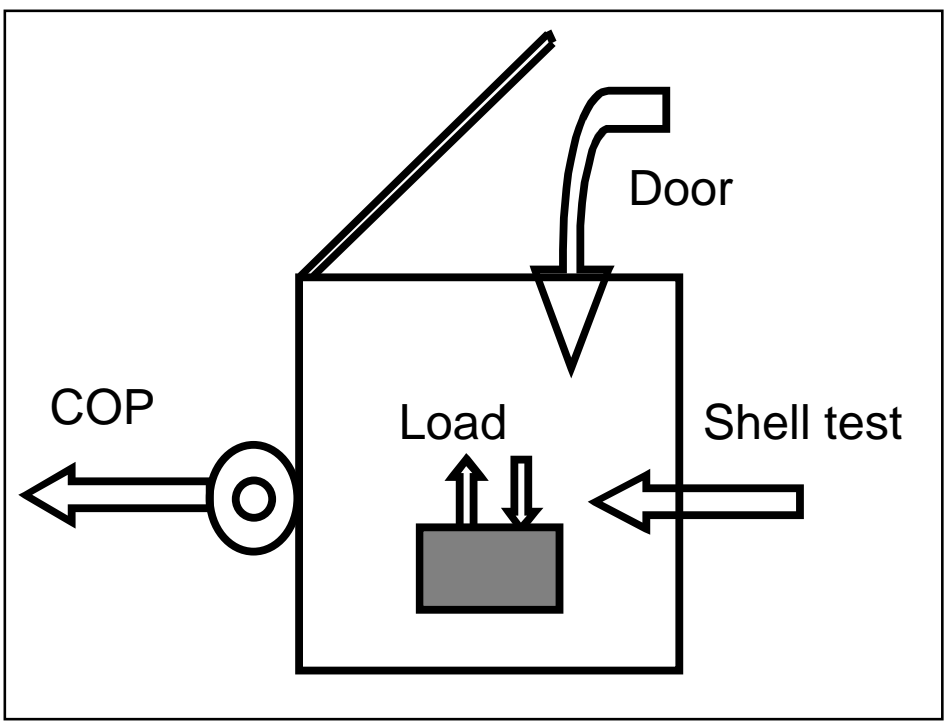

Figure 2. Major parameters in the refrigerator hardware tests.

model the appliance's performance accurately over a wide range of conditions.

The hardware test proposed to measure the refrigerator's efficiency of heat extraction (COP test) illustrates our attempts to satisfy the need for simple tests and performance data over a wide range of conditions. The goal is to measure the COP from part-load to full-load and at different ambient temperatures. In addition, the test must recognize that future compressors are likely to operate at different speeds or capacities. The test requires the placement of a heater that can be remotely-controlled inside the refrigerator. The test consists of the following steps:

1. Place the refrigerator in a test chamber at a specified ambient temperature and lower the inside temperature until the compressor must cycle to maintain the desired temperature.

2. The test begins when the compressor on-time is much less than the compressor off-time.

3. The heater is switched on at low power, and the refrigerator's temperature is allowed to stabilize at the original temperature.

4. The power of the heater is increased in small steps, each time waiting for temperature stabilization to be achieved. Intervals between cycling become gradually shorter.

5. The power is increased until the compressor is no longer able to maintain the desired inside temperature-even when the compressor is operating constantly-and the inside temperature begins to rise.

6. Repeat steps $1-5$ at a second ambient temperature. 
The results of one series of tests are illustrated in Figure 3. The left chart shows the incremental increases in heater power over time. The right chart shows the internal

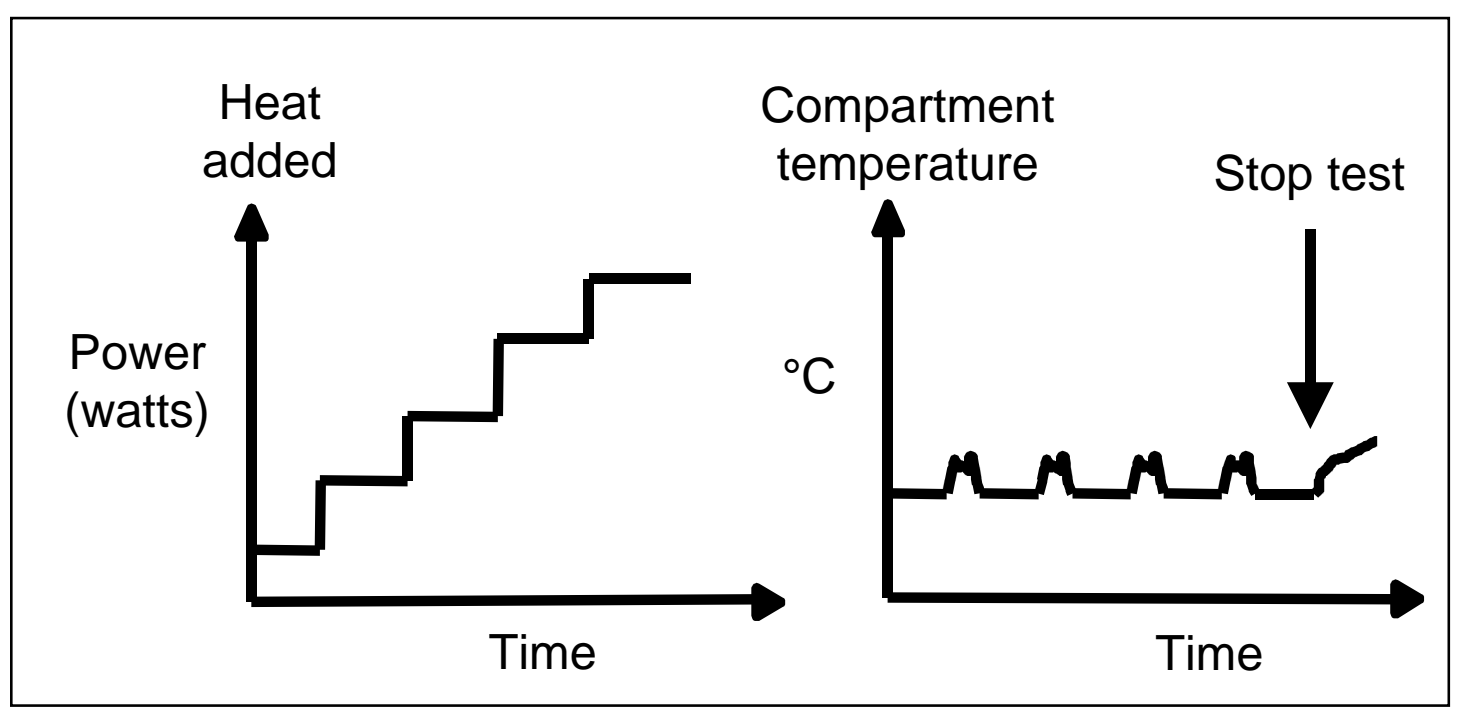

Figure 3. Hardware test to measure COPs.

temperature at each level of heat input. The temperature fluctuates a little after each increase but then returns to the thermostat setting. When the compressor's heat removal capacity is finally overwhelmed, the inside temperature climbs above the thermostat setting.

These measurements are sufficient to develop a performance curve (as shown in Figure 4) for the compressor system. The surface captures efficiency at both partload conditions and at two ambient temperatures. Interpolation to other temperatures is also possible without significant loss of accuracy. These performance curves will be used by the simulation model to predict energy consumption of the tested unit over a broad range of conditions.

We have developed hardware tests for measuring heat loss, door-opening, and loads, but there is not enough space here to describe them and are reported elsewhere (Wihlborg and Ernebrant 1999). Most of the tests could be easily automated, so they need not necessarily be more complicated to perform. Some have already been done and reported in the literature (Alissi, Ramadhyani et al. 1988).

\section{Software Tests}

The goal of the software test is to evaluate the "intelligence" of the microprocessor. How does it respond to different situations? Are its algorithms crude or sophisticated? Are there certain combinations of conditions where the appliance behaves erratically? Are the algorithms consistent with the appliance's mechanical parameters? 


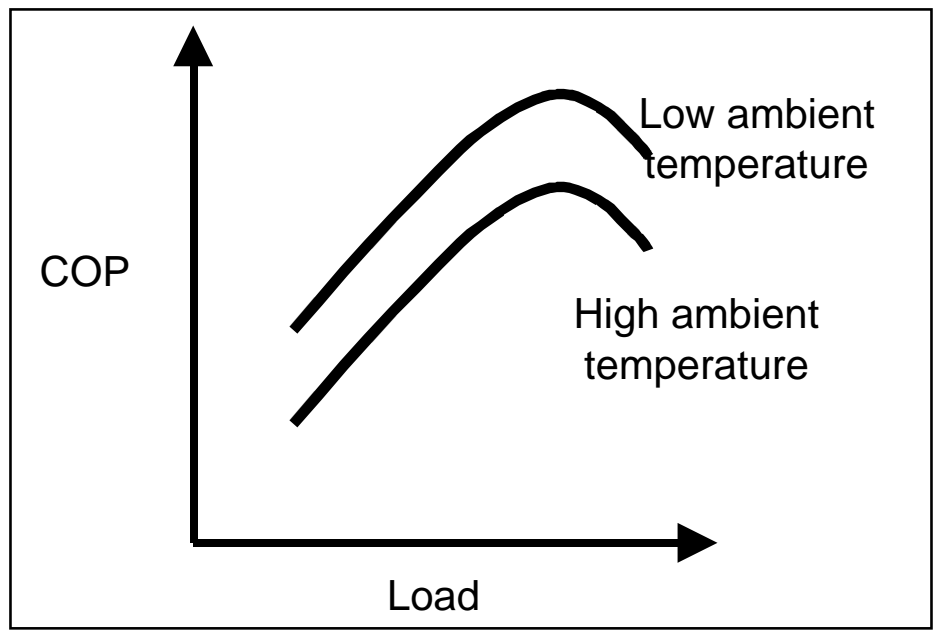

Figure 4. Derived COP parameters

We assume that the microprocessor can be interrogated, either in place via a communications cable, or extracted and tested on the bench. Even though this feature is not now generally available, some manufacturers already have installed this capability to interrogate the microprocessor in order to diagnose technical problems. (One manufacturer uses the communication to erase the microprocessor's memory of previous cycles in order to ensure consistent testing.) Microprocessors can already be removed in most cars and some dishwashers (because manufacturers expect to change the cleaning algorithms when new detergents are introduced). Manufacturers are also creating "network ready" appliances which assume communications ability.

Our approach to assessing the appliance's microprocessor relies on a form of reverse engineering. A computer creates thousands of different combinations of conditions. It submits them to the appliance microprocessor (as if these were the inputs from the controls and sensors) and records the microprocessor's responses. The computer assembles the microprocessor's replies into "response surfaces." An example of a 2dimensional hypothetical response surface is shown in Figure 5.

The response surfaces are converted into equations and passed to the simulation model. These equations will be used to control the appliance's operation.

Response surfaces may be relatively simple for most of today's appliances because the microprocessor typically controls one or two aspects of the appliance. For example, microprocessors in many refrigerators control the defrost interval and data displayed on a small screen. Future response surfaces will become very complex when the microprocessor controls several functions (such as defrost interval, compressor speed, and temperature distribution in a refrigerator) and relies on more than a few sensors. Microprocessors employing fuzzy logic, where their response 
depends on earlier conditions, add yet another layer of complexity. Most defrost controls remember only a few defrost cycles but at least one manufacturer tracks the last ten cycles.

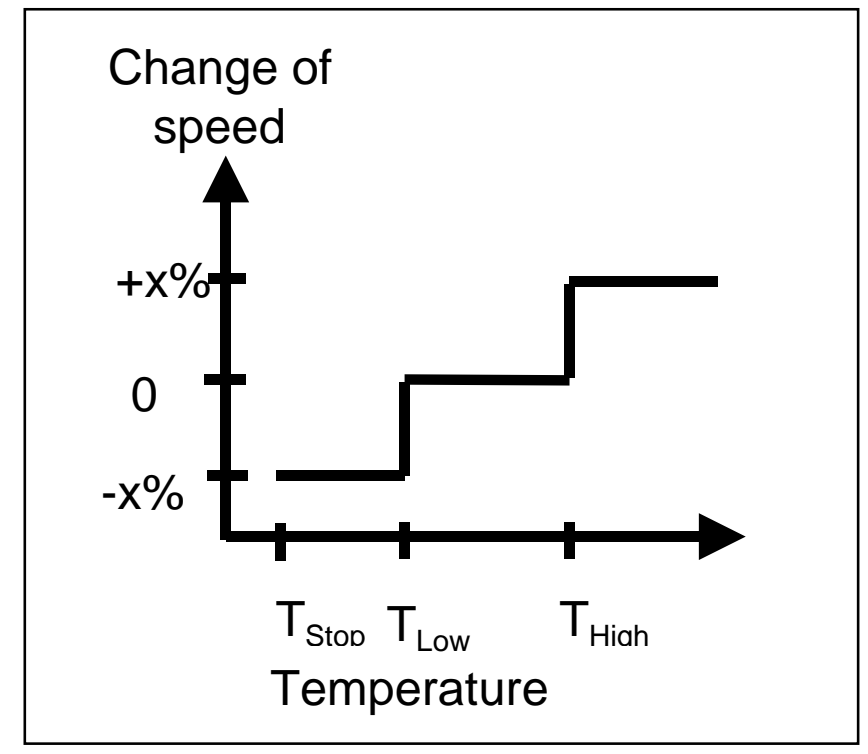

Figure 5. A hypothetical response surface for a variable speed motor.

We anticipate that the microprocessor will need to be interrogated millions of times in order to develop smooth response surfaces. This can nevertheless be accomplished in a reasonably short time because the interrogations require very small fractions of a second.

\section{Simulation Model and Outputs}

The third element in the test procedure is a dynamic simulation model of the appliance. This model simulates the operation of the appliance and predicts its energy consumption under specified conditions. The specified conditions may be those specified by the ISO, DOE, or JIS tests or any other conditions.

Simulation models for several appliances have already been developed for refrigerators (Arthur D. Little Inc. 1982), air conditioners, furnaces, and water heaters (Hiller and Lowenstein 1992; Paul and Whitacre 1993). Most of these models assume steady-state behavior; these are not realistic when trying to simulate the decisions of the microprocessor, which are dynamic. Recently, two water heater models have been developed that simulate dynamic aspects, such as hot water drawdowns, the recovery period, and thermal stratification. These models resemble those used to simulate building energy loads, in which the materials, their physical properties, and geometry are all specified. 
We also use a dynamic model but employ a different approach for specification of the appliance. Our model relies on lumped parameters instead of detailed specifications of all of the physical characteristics of the materials and components. The model is very general and relies on inputs from the hardware and software tests to give it structure and uniqueness. Complex, dynamic models are now reasonably easy to construct using commercially available software, so models for other appliances could be made.

The software and hardware tests must be carefully coordinated with the simulation model. The challenge is to develop hardware and software tests that provide suitable parameters for use by the simulation model. We found that for the refrigerator model the approach to simulation depended upon the kinds of data available from the hardware and software tests. Likewise, we revised the hardware tests several times in order to provide the most useful information to the model. In other words, an iterative approach is most successful.

The model simulates the appliance's operation and estimates its energy consumption. The model requires a detailed description of the hypothetical schedule and conditions, that is, the period of measurement, the ambient temperature, inside temperatures, humidity, number of door openings, etc. For example, the DOE refrigerator test specifies that the refrigerator be tested at ambient temperature of $32^{\circ} \mathrm{C}$ for 24 hours, or from defrost to defrost. The JIS refrigerator test includes door openings with a specific schedule.

We successfully simulated the energy use of the same refrigerator when tested according to the ISO, DOE, and JIS test conditions. Thus, we were able to prepare energy consumption estimates (such as those needed for energy labels or regulatory purposes) for three different markets using a single test procedure.

\section{CONCLUSIONS}

We have presented a framework for a new energy test procedure. It addresses a major flaw in current test procedures, that is, they test only the mechanical aspects of the appliances and ignore software aspects. Microprocessor control (coupled to extensive use of sensors) is likely to save significantly more energy than mechanical improvements in the next decade, so it is important to capture those benefits.

Our approach also offers a novel solution to the harmonization problem. In the case of our approach, everyone can agree on the same hardware tests, software tests, and simulation model. Each country may select its own output from the model. This output (which might appear on its energy-use labels, or be part of its minimum efficiency regulations) would reflect the unique conditions faced there.

Our approach is considerably more complicated than current test procedures. This is not surprising, because it seeks to capture the energy impacts of complex interactions 
between mechanical and controls aspects of the appliance. Furthermore, we have not demonstrated that the hardware and software tests for one appliance are technically feasible and that a model can simulate all the key aspects of operation. Nevertheless, we have shown how the parts fit together, and we have shown potential benefits from the proposed test procedure. We plan to refine the test and apply it to a real refrigerator soon. In the meantime, we invite your comments and suggestions.

\section{ACKNOWLEDGEMENTS}

This work was supported by the Assistant Secretary for Energy Efficiency and Renewable Energy, Building Technologies, of the U.S. Department of Energy under Contract No. DE-AC03-76SF00098.

\section{REFERENCES}

Alissi, M.S., S. Ramadhyani and R.J. Schoenhals 1988. "Effects of Ambient Temperature, Ambient Humidity, and Door Openings on Energy Consumption of a Household Refrigerator-Freezer." ASHRAE Transactions 94(2): 1713-1735.

Arthur D. Little Inc. 1982. Refrigerator and Freezer Computer Model User's Guide. Cambridge (Mass.): Arthur D. Little Inc.

Domanski, P.A. 1989. Rating Procedure for Mixed Air-Source Unitary Air Conditioners and Heat Pumps Operating in the Cooling Mode - Revision 1. NISTIR 89-4071. National Institute of Standards and Technology.

Hiller, C. and A Lowenstein. 1992. WATSIM User's Manual, Version 1.0: EPRI Detailed Water Heating Simulation Model User's Manual. Palo Alto (Calif.): Electric Power Research Institute.

Japanese Standards Association. 1979. Household Electric Refrigerators, Refrigerator-Freezers and Freezers, Japanese Industrial Standard JIS C 9607. Tokyo: Japanese Standards Association.

Meier, Alan. 1998. "Energy Test Procedures for the Twenty-First Century" In the Proceedings of 1998 Appliance Manufacturer Conference and Expo. Nashville, Tenn.: Appliance Manufacturer Magazine.

Meier, Alan K. 1997. "The Next Generation of Energy Test Procedures" In the Proceedings of Proc. of First International Conference on Energy Efficiency in Household Appliances. Florence, Italy: Springer Verlag. 
Paul, D. and G. Whitacre. 1993. TANK Computer Program User's Manual with Diskettes: An Interactive Personal Computer Program to Aid in the Design and Analysis of Storage-Type Water Heaters. Columbus (Ohio): Battelle Memorial Institute.

Wihlborg, Mats and Stefan Ernebrant 1999. "Energy Test Procedures for Appliances". Department of Engineering. Lund (Sweden), University of Lund.

\section{Suggested citation for this paper:}

Meier, Alan. 2000. "A General Design for Energy Test Procedures" In the Proceedings of The Second International Conference on Energy Efficiency in Household Appliances. Naples (Italy): Association of Italian Energy Economics (Rome). 